\title{
Static vs dynamic FRR sizing for power systems with increasing amounts of renewables
}

\author{
M-L. Cauwet, E. Karangelos, L. Wehenkel \\ Montefiore Institute, Department of EE\& CS \\ University of Liège \\ Liège, Belgium \\ marieliesse.cauwet@gmail.com, \{e.karangelos - 1.wehenkel\}@uliege.be
}

\author{
B. Georis \\ Global Energy Managements \\ Engie \\ Brussels, Belgium \\ Bruno.georis@engie.com
}

\begin{abstract}
This paper investigates the sizing of the Frequency Restoration Reserve (FRR) in a context of increasing penetration of renewable generation. More precisely, we propose (i) a probabilistic method that mimics the current Belgian TSO (Elia) practices and (ii) a Monte-Carlo based procedure that evaluates the corresponding reliability of the system in terms of down/upward reserves activation, wind curtailment and load shedding. Using this method over the IEEE-RTS96 testcase, the impact of wind penetration - low, moderate, high - is studied. In particular, static (annual and seasonal) and dynamic (weekly and hourly) FRR sizing approaches are defined and compared. It turns out that the hourly sizing method is the most robust. It also appears that FRR requirements for upward reserves are almost not impacted by the high wind penetration whereas the downward reserves increase significantly with the wind penetration. Our implementations rely on Julia, Cplex and $\mathbf{R}$ and are available in open source.
\end{abstract}

Index Terms-Power system reliability, renewable integration, probabilistic reserve capacity dimensioning, Monte-Carlo methods.

\section{INTRODUCTION}

In power systems operation, Frequency Restoration Reserves (FRR) are needed in order to ensure, in spite of uncertainties, equilibrium between generation and demand in a given area at any moment, and to contribute in a proper way to stabilize the frequency in the interconnected system. If the amount of available reserves at some moment in time is too small, energy supply is at risk and load-shedding or curtailment of renewable energy sources may have to be used as last resort actions. On the other hand, if the amount of reserves is often too high, it means that the system is underutilized and hence sub-optimal from an economic point of view.

More renewable generation leads to both more variability and more stochasticity in the net demand (actual demand minus renewable generation). In order to cope with these trends, increased amounts of FRR may be needed and hence increased costs may be foreseen. In most places, as in Belgium [1], the requirements of different kinds of FRR are currently defined in a static fashion on a yearly basis (same requirement for every hour of the next year).

A possible direction of improvement could be to define the required amounts of reserves in a more dynamical (i.e., timevarying) fashion, thereby allowing the TSO to more effectively adapt the amount of purchased reserves to the actual needs at any given moment of time. Indeed, this could allow to improve the overall social welfare by balancing in a more efficient way the impact of reserve sizes on energy prices and on reliability.

The paper's contribution has a twofold purpose. We first propose a methodology that enables to estimate the FRR needs taking into account the probability of an imbalance between generation and demand. This method is close to the actual TSOs practises, which gradually move from the traditional $N-1$ criterion toward probabilistic sizing. Together with this FRR sizing method, we propose a reliability evaluation of the resulting system in terms of probability of being short in upward and downward reserves, expected amount of demand not served and of wind curtailment and expected amount of upward and downward reserve activation. These performances are established by Monte-Carlo simulations. Second, using this method on the IEEE-RTS96 modified to include renewable generations, we analyse how the FRR size evolves (i) in front of low, moderate and high wind penetration (ii) when being fixed for one hour, one week, one season or one year and what is the impact on the system's reliability.

The remainder of the paper is organized as follows. Section II describes the probabilistic methodology used to compute the requested FRR dimension and the reliability evaluation procedure. Section III presents an empirical study on the IEEE-RTS96 benchmark, where the three areas have to cope with different levels of wind-power penetration, from very high in the West, to moderate in the East. Section IV discusses related work and Section V concludes and identifies several directions of further research.

\section{FRR SIZING \& EVALUATION METHODOLOGY}

We consider a probabilistic reserve sizing method similar to the one defined by ENTSOe. This method requires that the probability that the level of reserve in a control area is not sufficient to cover the imbalances of this area should not exceed $0.1 \%$ [2]. Hence the first step is to obtain the distribution function of the imbalances. For this purpose, we model the sources of imbalance in Section II-A. In this paper, the sources of imbalances are (i) the generation unit outages; (ii) the difference between the day-ahead load forecast and its realization; (iii) the difference between the day-ahead 
wind generation forecast and its realization. The sources of imbalances are considered as independent.

\section{A. Physical and uncertainty models}

1) Conventional generation unit outages: The probability $P$ that an unplanned outage occurs during a time interval $[t, t+\Delta t]$ depends only on $\Delta t$ and on the failure rate $\lambda$. It is modeled by an exponential distribution [3]:

$$
P(\Delta t)=1-\exp (-\lambda \cdot \Delta t) .
$$

In order to compute the probability distribution of imbalances due to outages of conventional generation units, we adopt the following assumptions, similar to [1]:

- a unit can be lost only once during the period $\Delta t=1 \mathrm{~h}$;

- after at most $1 h$, any unit will be back online;

- outages of different units are independent;

- in case of outage occurence, it is assumed that the unit has been dispatched at its maximum output;

- only thermal and nuclear are considered.

The probability distribution of the loss capacity in the production park is then computed, by convolution, using (1) and the assumptions above.

For the numerical study, the failure rate of the generating units is taken from the literature [4].

2) Load and wind generation forecast error: Demand and renewable stochasticities at a time step $t$ are modelled as truncated Gaussian white-noise relative deviations from dayahead forecast. Hence the load realization $p_{\text {real }}^{t}$ is given by:

$$
p_{\text {real }}^{t}=p_{d a}^{t}(1+\mathcal{N}(\sigma)),
$$

where $p_{d a}^{t}$ is the load forecast and $\mathcal{N}(\sigma)$ is a centered truncated Gaussian of standard deviation $\sigma$ and lying in an interval $[-\eta, \eta]$.

Equation (2) gives directly the load error distribution for a time step $t$. We can derive the load error distribution over a period of $T$ time steps by:

$$
\begin{aligned}
\mathbb{P}\left(p_{\text {real }}^{t}-p_{d a}^{t}>x\right) & =\sum_{s=1}^{T} \mathbb{P}\left(p_{\text {real }}^{t}-p_{d a}^{t}>x \mid t=s\right) \mathbb{P}(t=s), \\
& =\frac{1}{T} \sum_{s=1}^{T} \mathbb{P}\left(p_{d a}^{s} \mathcal{N}(\sigma)>x\right) .
\end{aligned}
$$

It is a mixture of truncated Gaussians. If $T=1$ the distribution is simply a truncated Gaussian.

For the numerical study, $\sigma$ and $\eta$ are taken to be consistent with the values of the 2016 Belgian grid. Note that these values can be found in the implementations available at [5].

The wind generation forecast error for each wind station is modeled similarly.

\section{B. Computation of the imbalances distribution}

The three previous distributions (outages, load and wind errors forecasts) are convoluted, in order to obtain the distribution of imbalances. Then, the upward reserve requirement is computed by taking the $99.9 \%$ quantile of the positive part of the imbalance distribution, and the downward reserve requirement is computed by taking the $0.1 \%$ quantile of the negative part of the imbalance distribution.

\section{Evaluating the actual impact of the reserve sizing policies}

In order to evaluate the resulting system, we propose an approach based on crude Monte-Carlo simulation. First, day-ahead unit generation scheduling together with the units providing the reserves are given by a joint unit commitment, assuming copper-plate network. The detailed unit commitment is provided in [5]. Then, some scenarios of outages and deviations of demand and wind-generation from forecasts, at an hourly time step, are generated by using the probabilistic model presented in Section II-A. These realizations are used to estimate the following reliability indicators: (i) actual probability of shortage of FRR; (ii) expected level of activation of FRR; (iii) expected amounts of load-shedding and wind curtailment.

In our assessment, we aimed at evaluating probabilities of rare events (probability of reserve shortage $\approx 0.1 \%$ and expectations of quantities, such as expected amount of shortage, having a high variance). In order to estimate these quantities with sufficient accuracy and thus to avoid any discussion about the estimated values, we have used a very large number of Monte-Carlo samples, namely $10^{8}$.

\section{CASE STUdy ON THE 3-AREA IEEE-RTS96}

The probabilistic sizing method is applied to the IEEERTS96, modified in order to include 19 wind farms: 9 in the western area (Area 1), 7 in the central one (Area 2) and 3 in the eastern one (Area 3) [6]. The period considered for the study covers weeks 1 (representative of winter), 9 (representative of spring/autumn) and 18 (representative of summer) of [4] with an annual load peak of $2850 \mathrm{MW}$. The wind scenarios have been provided by [6]

In our study, we consider that each area should cover the imbalances originating from the generating sources and loads connected in that area. Using reserves from another control area would imply additional reliability considerations related to the cross-border transmission capacities that are out of the scope of the present study.

All the data necessary to reproduce these experiments can be found in [5].

\section{A. Reserves sizes}

Tables I and II display the average upward and downward FRR sizes resulting from the probabilistic methodology for each case. The annual static case is constant over the whole period of 3 representative weeks (i.e., $T=504$ in (4)), while the weekly reserve requirement is adjusted for each week (i.e., $T=168$ in (4)). These weekly reserves requirement are presented in Tables III and IV. Going down to daily (i.e., $T=24$ in (4)) and hourly (i.e., $T=1$ in (4)) allocations, Fig. 1 and 2 show the variations of the corresponding reserves requirements. 
TABLE I: Average upward reserves (in MW).

\begin{tabular}{|l|r|r|r|}
\hline & Area 1 & Area 2 & Area 3 \\
\hline \hline Annual & 524.3 & 482.7 & 425.9 \\
\hline Weekly & 525.2 & 481.2 & 426.1 \\
\hline Daily & 523.2 & 480.2 & 426.3 \\
\hline Hourly & 520.4 & 480.0 & 428.6 \\
\hline
\end{tabular}

TABLE II: Average downward reserves (in MW)

\begin{tabular}{|l||c|c|c|}
\hline & Area 1 & Area 2 & Area 3 \\
\hline \hline Annual & -443.2 & -350.5 & -135.0 \\
\hline Weekly & -417.3 & -322.8 & -125.9 \\
\hline Daily & -391.7 & -305.2 & -116.1 \\
\hline Hourly & -350.4 & -274.8 & -106.8 \\
\hline
\end{tabular}

1) Impact of the wind penetration: Both upward and downward reserves are higher for the areas having a higher penetration of wind-power. This dependance is however much stronger for the downward reserves, which do not have to cover the conventional generating units outages (the latter having only an impact on the upward reserve requirements and being in our study modeled identically in the three areas). Daily and hourly variations of reserves requirement are most important in area 1, where the wind penetration is the largest.

2) Impact of the reserve size temporal variations: The seasonal, daily and hourly sizing methods yield reserve requirements that indeed vary significantly from one season to another, from day to day, and from hour to hour. On average over the three study weeks, the upwards reserve requirements are however almost not affected, but the downwards reserve requirements decrease significantly, the effect being stronger in the more wind-intense areas and for the more dynamic methods (reduction of $20 \%$ in the western area, when using the hourly sizing method, reduction of $9 \%$ in the eastern area,

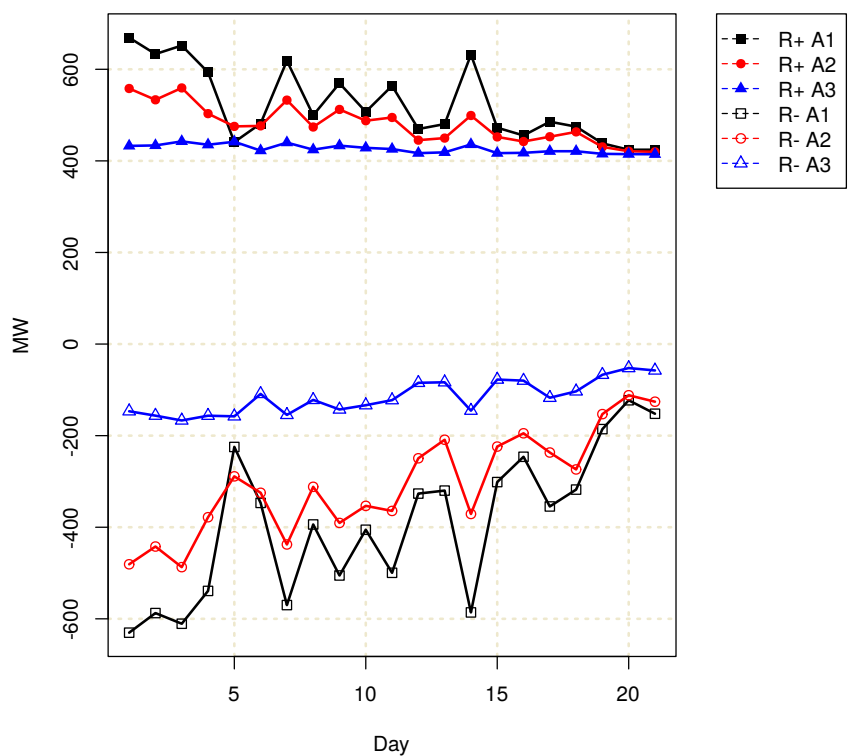

Figure 1: Daily probabilistic reserve sizing (in MW). Areas A1, A2, A3 are ordered from West to East, i.e., from high to low wind penetration. 'R+' (resp. 'R-') stands for upward (resp. downward) reserves.
TABLE III: Seasonal upward reserves (in MW).

\begin{tabular}{|r|r|r|r|}
\hline & Area 1 & Area 2 & Area 3 \\
\hline \hline Week 1 (winter) & 588.6 & 520.8 & 435.3 \\
\hline Week 9 (spring) & 531.7 & 481.7 & 426.1 \\
\hline Week 18 (summer) & 455.5 & 441.2 & 417.0 \\
\hline
\end{tabular}

TABLE IV: Seasonal downward reserves (in MW).

\begin{tabular}{|c|c|c|c|}
\hline & Area 1 & Area 2 & Area 3 \\
\hline \hline Week 1 (winter) & -533.7 & -424.0 & -155.1 \\
\hline Week 9 (spring) & -451.5 & -336.1 & -127.6 \\
\hline Week 18 (summer) & -266.7 & -208.3 & -95.1 \\
\hline
\end{tabular}

when using the seasonal sizing method).

3) Discussion: From the above results, we may be tempted to conclude that (i) the seasonal, daily and hourly variations of load and wind-generation profiles could be exploited in order to more dynamically adjust reserve requirements in such a way that the overall reserve requirements are reduced significantly for downward reserves and more marginally for upward reserves, and (ii) that the expected potential for such dynamic reserve sizing methods should increase with higher levels of wind-generation penetration.

This possibility should however be confronted with the fact that energy market prices themselves are as well impacted by the seasonal, daily and hourly dynamics of load and renewable generation, so that at the moments when more reserve is required, the impact of purchasing more of it on these latter energy markets would be amplified, and in the end be possibly counter-productive from an overall socio-economic viewpoint.

On the other hand, we would like to stress the fact that the strategies investigated above in order to adjust reserve requirements are by no means optimal, since they are still computed in foresight based on seasonal, daily and hourly

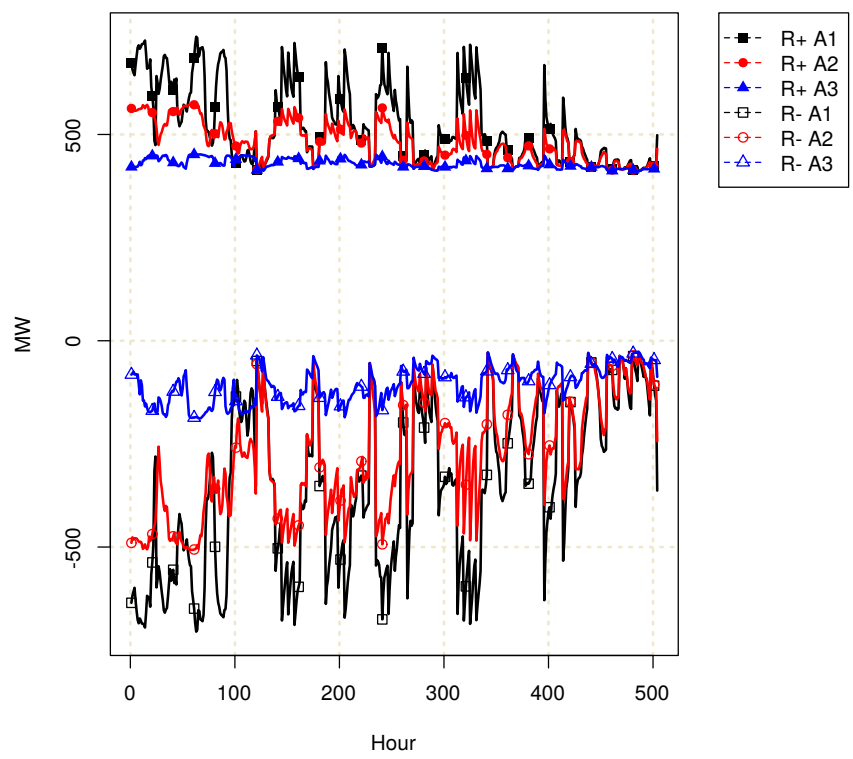

Figure 2: Hourly probabilistic reserve sizing (in MW). Areas A1, A2, A3 are ordered from West to East, i.e., from high to low wind penetration. 'R+' (resp. 'R-') stands for upward (resp. downward) reserves. 
TABLE V: Estimated actual probability (in \%) that the required reserve level is sufficient to cover the imbalance. We provide a large number of digits to facilitate verification in the context of replication studies.

\begin{tabular}{|c|c|c|c|}
\hline & Area 1 & Area 2 & Area 3 \\
\hline 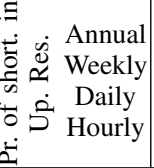 & $\begin{array}{l}99.95284 \pm 10^{-3} \\
99.97001 \pm 10^{-2} \\
99.97450 \pm 10^{-3} \\
99.97370 \pm 10^{-3}\end{array}$ & $\begin{array}{c}\mathbf{9 9 . 8 7 1 4 8} \pm \mathbf{1 0}^{-2} \\
99.81499 \pm 10^{-2} \\
99.83044 \pm 10^{-2} \\
99.84596 \pm 10^{-3}\end{array}$ & $\begin{array}{l}99.89655 \pm 10^{-3} \\
99.89732 \pm 10^{-3} \\
99.89056 \pm 10^{-3} \\
99.89588 \pm 10^{-3}\end{array}$ \\
\hline 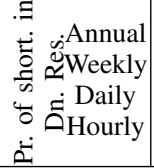 & $\begin{array}{c}99.87224 \pm 10^{-2} \\
99.83007 \pm 10^{-2} \\
99.85437 \pm 10^{-2} \\
\mathbf{9 9 . 9 7 4 1 4} \pm \mathbf{1 0}^{-3}\end{array}$ & $\begin{array}{c}99.90943 \pm 10^{-2} \\
99.95286 \pm 10^{-2} \\
99.95899 \pm 10^{-2} \\
\mathbf{9 9 . 9 9 3 2 8} \pm \mathbf{1 0}^{-3}\end{array}$ & $\begin{array}{c}99.87865 \pm 10^{-2} \\
99.92270 \pm 10^{-2} \\
99.94504 \pm 10^{-2} \\
\mathbf{9 9 . 9 9 5 1 6} \pm \mathbf{1 0}^{-3}\end{array}$ \\
\hline
\end{tabular}

statistics available in advance (and hence do not adjust to incoming information), and since they were designed so as to maintain the probability of shortage constant and equal to the $99.9 \%$ requirement over each sub-period considered; better strategies could indeed possibly take advantage of incoming information and a degree of freedom for allocating the risk of shortage itself in a dynamic fashion.

\section{B. Reliability assessment}

This section presents and analyses the resulting system reliability, evaluated using the method described in Section II-C. Note that extended results are available in [5]. In the following, the $99.9 \%$ confidence interval is given after the symbol ' \pm '.

Probability of being short in upward reserve - Area 1

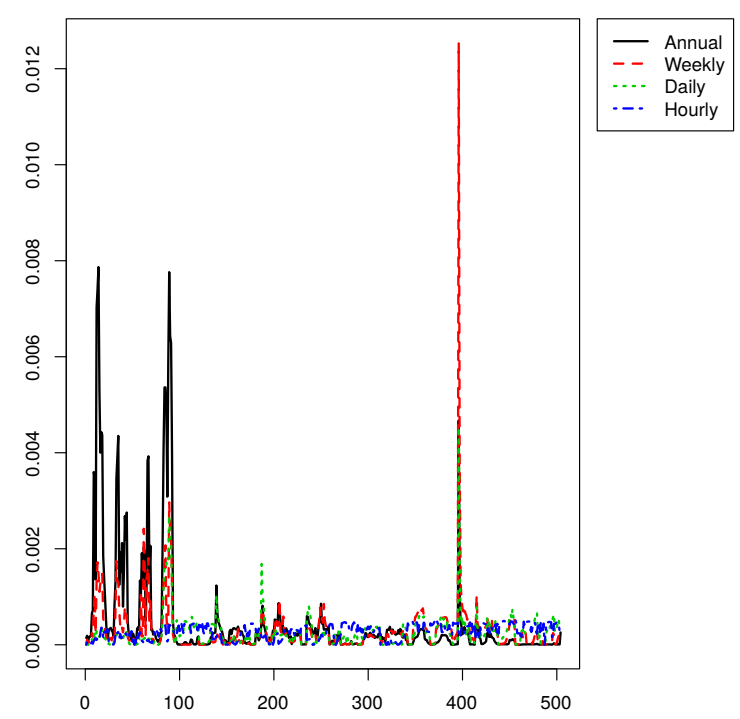

Figure 3: Probability of being short in upward reserve in Area 1, estimated by Monte-Carlo over $10^{8}$ samplings, $99.9 \%$ confidence interval in $\pm 10^{-5}$ (resp $\pm 10^{-4}, \pm 10^{-5}, \pm 10^{-5}$ ) for the 'annual' (resp. 'weekly', 'daily', 'hourly') setting.

For all three areas, and for all four FRR sizing methods, an average reliability level of about $99.8 \%$ is indeed reached when integrating over the whole study period, see details in
TABLE VI: Average upward and downward activated reserves over the whole period (in $\mathrm{MWh} / \mathrm{hr}$ ).

\begin{tabular}{|c|c|c|c|}
\hline & Area 1 & Area 2 & Area 3 \\
\hline 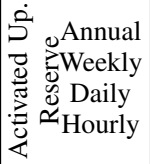 & $\begin{array}{l}40.58108 \pm 10^{-1} \\
40.25705 \pm 10^{-1} \\
39.27389 \pm 10^{-1} \\
39.08801 \pm 10^{-1}\end{array}$ & $\begin{array}{l}103.7042 \pm 10^{-1} \\
106.3275 \pm 10^{-1} \\
109.8709 \pm 10^{-1} \\
111.1260 \pm 10^{-1}\end{array}$ & $\begin{array}{l}89.60536 \pm 10^{-2} \\
90.98905 \pm 10^{-2} \\
95.24042 \pm 10^{-2} \\
96.63406 \pm 10^{-2}\end{array}$ \\
\hline 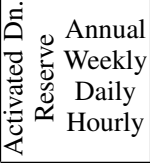 & $\begin{array}{l}40.35395 \pm 10^{-1} \\
40.01484 \pm 10^{-1} \\
39.07639 \pm 10^{-1} \\
38.92324 \pm 10^{-1}\end{array}$ & $\mid \begin{array}{l}23.16114 \pm 10^{-1} \\
22.71596 \pm 10^{-1} \\
22.15356 \pm 10^{-1} \\
21.63147 \pm 10^{-1}\end{array}$ & $\begin{array}{l}8.649286 \pm 10^{-2} \\
8.660424 \pm 10^{-2} \\
8.180613 \pm 10^{-2} \\
7.627230 \pm 10^{-2}\end{array}$ \\
\hline
\end{tabular}

Table V. However, as shown on Fig. 3 and 4 for the western area, the annual, weekly and daily reserve sizing methods lead to variations of the probability of shortage from hour to hour, with peaks in the more difficult days and hours that can be significantly higher than the set targets.

As concerns the level of reserve activation, we observe in Table VI no significant differences among the different sizing methods when integrating over the study period. These levels are however quite different from one area to the other. For example, in the western area, average upward and downwards activation levels are of similar magnitude and represent about $10 \%$ of the FRR reservation. In the eastern area, on the other hand, upwards reserve activation is about ten times higher on average than the average downwards activation. The more wind-intensitive area presents a symmetry in terms of upward and downward reserves activation, i.e., the predominant factor

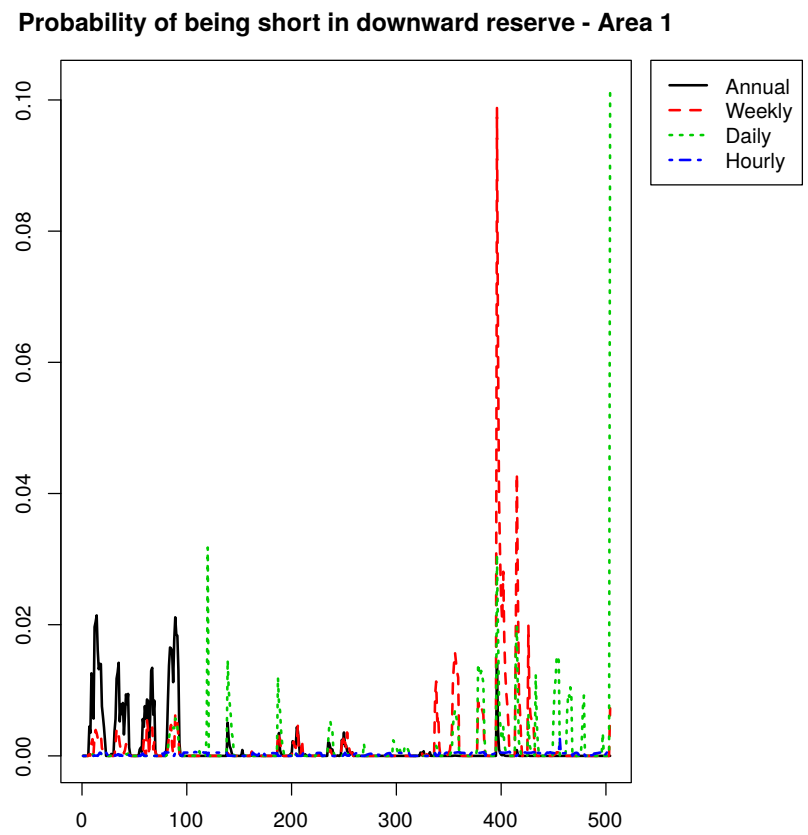

Figure 4: Probability of being short in downward reserve in Area 1, estimated by Monte-Carlo over $10^{8}$ samplings, $99.9 \%$ confidence interval in $\pm 10^{-4}$ (resp. $\pm 10^{-4}, \pm 10^{-4}, \pm 10^{-5}$ ) for the 'annual' (resp. 'weekly', 'daily', 'hourly') setting. 
of imbalance is the wind uncertainty, and not anymore the units outages. Overall, we observe that average activation levels of reserves are quite lower than the amounts prescribed by the different reservation policies.

\section{RELATED WORK}

\section{A. FRR dimensioning methods}

A large span of the literature is devoted to the modeling of the sources of imbalances by analysing historical data. It is often proposed to first estimate the distributions of each driver of the imbalances and then to do the convolution of these distributions assuming that they are not correlated [7], [8]. Regarding to the type of distribution to select, we refer to the survey of [9], to [7], which investigates the use of Kernel distributions, and [10] for the wind error distribution. The main limits of this method is the handling of correlations between the different sources of imbalances and the distributions adjustment. Another approach relies on machine learning method, which is used for features selection and reserves sizing. Regarding feature selection, a preliminary work has been done in [11], using k-Nearest Neighbor method, Random Forest, Gradient boosting Tree and Principal Component Analysis. The study emphasises that the sources of imbalances do not have the same impact within different control areas (TenneT, Amprion, 50Hertz), e.g., the wind generation has a lower impact in the Amprion control area than in the two others. [12] uses the Lasso to weight the sources of imbalances, which can be detrimental if two variables are highly correlated. As for reserve needs prediction, [13], [14] study the case of the German control area for a quarter of hour time step, using a neural network and quantile regression. A key issue in this work was the sparse data regarding the power unit outages, requiring an adaptive bias correction function. Reference [12] studies the case of the Austrian control area for a day-ahead reserve dimensioning, using the Lasso and quantile regression.

Overall, these studies emphasize the good performance of dynamic dimensioning. However, most of them investigate only the adequacy between prediction and realization of the imbalances and do not consider the problem in terms of power system costs. The following paragraph considers contributions on reserve from an economical viewpoint.

\section{B. FRR costs}

Regarding the procurement of reserves, [15] presents a two-stage stochastic market-clearing model to determine the amount of reserves that should be procured in each market stage (weekly and daily auctions) in order to minimizes the expected procurement costs of reserves, while taking reserve dimensioning criteria and market properties into consideration. This model is clearing the reserve market in Switzerland since 2014. Reference [7] uses the Joint Market Model to quantify the reserve costs of dynamic and static reserve, where the reserve capacity prices are deduced as shadow prices from the constraints which stipulate that reserves must be met. The study on a 2030 German case emphasises a more efficient provision of reserves through dynamic reserve sizing (lower reserve and spot prices on average). The increased economic efficiency (more than 10M euros) was in favor of the consumers while the producers were exposed to financial losses. Reference [16] evaluates how participating in a reserve capacity market affects a hydro power producers decisions compared to only participating in the day-ahead market. A multi-stage model that is able to bids into multiple sequential markets is developed. The case study considers a Norwegian hydro power plant over a frametime of one week. The results show that participating in the reserve capacity market is most profitable when day-ahead prices and reservoir levels are low. Reference [17] investigates the benefit of coordinating the sizing, allocation and activation of reserves among market zones, using a unit commitment model and dispatch decisions. Though such coordination can lower operational costs and increase system reliability, in this model it turns out that decoupling sizing and allocation and only coordinating activation is the optimal strategy, because it neglects transmission constraints at the sizing and allocation stages.

In these studies, apart for [7] and [17], the reserve requirement is an input of the problem and hence, the potential cost due to an over or under-sizing of the FRR is not included.

The present paper is a first step toward covering simultaneously the 2 above aspects: FRR sizing and evaluation in term of impact on the system.

\section{CONClusion}

This paper presents a methodology to compute the FRR size in a probabilistic manner and evaluate it, complying with the real-word practices. This method has been tested on the IEEE-RTS96 three area system, where the different areas are subject to different levels of renewable generation, in this case represented as wind-farms. On this study, we have exemplified how reserve requirements could be temporally adapted. Finally, we have shown how reserve dimensioning policies could be evaluated from a reliability point of view, by applying a Monte-Carlo simulation approach and discussing the resulting performance indicators.

The present study can be viewed as a template of how different approaches for defining active power reserves can be studied, evaluated, and hence optimized.

\section{A. Discussion of the assumptions}

Several simplifying assumptions have been made in the models. We first notice that the outage capacity distribution computed for conventional generators is in some sense a worstcase distribution, since it assumes that all installed generating units in a given area are (always) on-line and operating at nominal power; on the other hand, the assumption that after an outage of one hour a unit capacity loss will be compensated by the generating company may be overly optimistic. In order to make the model more realistic, more information about conventional units dispatch statistics and repair times would be required.

Concerning the load-forecast errors, we believe that the proposed model is suitable, but we have chosen its parameters 
in an ad hoc way to fit public information about global load-forecast errors. In practice, these parameters should be correctly tuned to the available statistics about a given system. Concerning the wind-generation forecast errors, we have assumed that they are independent for different wind-farms, and independent of the load-forecast errors; both assumptions are to be validated based on observational data, specially if the net load (as it is modelled at the EHV level) incorporates a significant part of dispersed photovoltaic sources.

Last, we did not include an uncertainty factor that takes into account the temporal distance between the time at which the sizing is computed and the time at which we have a dayahead forecast. That is, the static methods (annual, weekly) have access to the exact day-ahead forecasts even 3 weeks or one week ahead. It is a strong, optimistic, assumption which allows us to decouple 2 effects:

- the impact of the forecast precision on the reserve size method;

- the time frame, or granularity, of a reserve size method.

We here study the impact of the latter on the power system reliability.

\section{B. Further work}

In order to complete the analysis of the possible impact of higher penetration of renewables on FRR reserve requirements several directions of further research have been identified.

1) PV penetration: a similar study should focus on the impact of PV penetration, first in isolation, then in conjunction with wind-power penetration. For this, suitable models or PV variability and uncertainty should be developed, since these are quite different from the behaviour of wind-power.

2) Manual vs automatic FRR: in addition to the distinction between upwards and downwards FRR requirements, it would be of interest to extend the model to take into account the distinction between manual and automatic FRR requirements.

3) Socio-economic analysis: in complement to the technical analysis provided in our study, it would be interesting to carry out an analysis to appraise the impact of FRR sizing methods and renewable penetration on the energy and reserve market prices, TSO costs, and costs of service interruptions (in terms of VOLL for the energy not served, and in terms of possible penalties for the curtailment of generation). Such a study would also allow to assess the relevance of the $0.1-99.9 \%$ reliability targets, in terms of costs and benefits.

4) More sophisticated FRR sizing methods: ideally, a reserve sizing method should target the maximization of the overall social welfare. Therefore other reserve sizing methods explicitly aiming at such a maximization could be proposed.

5) More accurate probabilistic models: with respect to the models used in our study several improvements could be imagined, such as more realistic thermal and hydro power-plant failure models, relaxing the gaussian white-noise assumption of various forecast errors, and more generally taking into account correlations among the different sources of imbalances.

\section{REFERENCES}

[1] ELIA, "Méthode d'évaluation et détermination de la puissance de réserve primaire, secondaire et tertiaire pour 2018," http://www.creg.be/sites/default/files/assets/Consult/2017/1631/89252-Art_233_Methode_et_Determination_devaluation_puissance_de_ reserve_2018_sans_TC.pdf, 2017, [Online; accessed 16-November2018].

[2] ENTSOe, "Continental europe operation handbook, policy 1: Loadfrequency control and performance," https://www.entsoe.eu/fileadmin/ user_upload/_library/publications/entsoe/Operation_Handbook/Policy_ 1_final.pdf, 2009, [Online; accessed 16-November-2018].

[3] R. Billinton, A. Bonaert, and A. Koivo, "Power system reliability evaluation," vol. 1, pp. 406 - 407, 111971.

[4] C. Grigg, P. Wong, P. Albrecht, R. Allan, M. Bhavaraju, R. Billinton, Q. Chen, C. Fong, S. Haddad, S. Kuruganty, W. Li, R. Mukerji, D. Patton, N. Rau, D. Reppen, A. Schneider, M. Shahidehpour, and C. Singh, "The IEEE reliability test system-1996. A report prepared by the reliability test system task force of the application of probability methods subcommittee," IEEE Transactions on Power Systems, vol. 14, no. 3, pp. 1010-1020, Aug 1999.

[5] M.-L. Cauwet, E. Karangelos, L. Wehenkel, and B. Georis, "Supplementary material for paper: Static vs dynamic FRR sizing for power systems with increasing amounts of renewables," 2018.

[6] H. Pandzic, Y. Dvorkin, T. Qiu, Y. Wang, and D. Kirschen, "Unit commitment under uncertainty - GAMS models, library of the Renewable Energy Analysis Lab (REAL), University of Washington, Seattle, USA,' http://www.ee.washington.edu/research/real/gams_code.html, [Online].

[7] M. Bucksteeg, L. Niesen, and C. Weber, "Impacts of dynamic probabilistic reserve sizing techniques on reserve requirements and system costs," IEEE Transactions on Sustainable Energy, vol. 7, no. 4, pp. 1408-1420, Oct 2016.

[8] D. Jost, A. Braun, and R. Fritz, "Sizing control reserves with a new dynamic method considering wind and photovoltaic power forecasts," in International Workshop on Large-Scale Integration of Wind Power into Power Systems as well as on Transmission Networks for Offshore Wind Power Plants, 21 - 13 November 2014, Berlin, Germany. Berlin, Germany: Springer, 2014.

[9] H. Holttinen, M. Milligan, E. Ela, N. Menemenlis, J. Dobschinski, B. Rawn, R. J. Bessa, D. Flynn, E. Gomez-Lazaro, and N. K. Detlefsen, "Methodologies to determine operating reserves due to increased wind power," IEEE Transactions on Sustainable Energy, vol. 3, no. 4, pp. 713-723, Oct 2012

[10] K. Bruninx, E. Delarue, and W. D'haeseleer, "Statistical description of the error on wind power forecasts via a Lévy $\alpha$-stable distribution," 2013, EUI RSCAS Working Paper 2013/50, 1-8.

[11] A. Ohsenbrügge and L. Sebastian, "Dynamic dimensioning of balancing power with flexible feature selection," in 23 rd International Conference on Electricity Distribution, June 2015.

[12] A. Essl, A. Ortner, R. Haas, and P. Hettegger, "Machine learning analysis for a flexibility energy approach towards renewable energy integration with dynamic forecasting of electricity balancing power," in 2017 14th International Conference on the European Energy Market (EEM), June 2017, pp. 1-6.

[13] D. Jost, A. Braun, and R. Fritz, "Dynamic dimensioning of frequency restoration reserve capacity based on quantile regression," in 2015 12th International Conference on the European Energy Market (EEM), May 2015, pp. 1-5.

[14] D. Jost, A. Braun, R. Fritz, and S. Otterson, "Dynamic sizing of automatic and manual frequency restoration reserves for different product lengths," in 2016 13th International Conference on the European Energy Market (EEM), June 2016, pp. 1-5.

[15] F. Abbaspourtorbati and M. Zima, "The swiss reserve market: stochastic programming in practice," IEEE Transactions on Power Systems, vol. 31, no. 2, pp. 1188-1194, 2016.

[16] E. S. Grytli, "Optimal bidding strategy in the reserve capacity market," Ph.D. dissertation, Norwegian University of Science and Technology, Norway, 2016.

[17] K. V. den Bergh, R. B. Hytowitz, K. Bruninx, E. Delarue, W. D'haeseleer, and B. F. Hobbs, "Benefits of coordinating sizing, allocation and activation of reserves among market zones," Electric Power Systems Research, vol. 143, no. Supplement C, pp. 140 - 148, 2017. [Online]. Available: http://www.sciencedirect.com/science/article/ pii/S0378779616304096 